\title{
Formal GAGA for good moduli spaces
}

\author{
Anton Geraschenko and David Zureick-Brown
}

\begin{abstract}
We prove formal GAGA for good moduli space morphisms under an assumption of "enough vector bundles" (which holds for instance for quotient stacks). This supports the philosophy that though they are non-separated, good moduli space morphisms largely behave like proper morphisms.
\end{abstract}

\section{Introduction}

Good moduli space morphisms are a common generalization of good quotients by linearly reductive group schemes [GIT94] and coarse moduli spaces of tame Artin stacks [AOV08, Definition 3.1].

Definition ([Alp09, Definition 4.1]). A quasi-compact and quasi-separated morphism of locally Noetherian algebraic stacks $\phi: \mathscr{X} \rightarrow \mathscr{Y}$ is a good moduli space morphism if

- $(\phi$ is Stein $)$ the morphism $\mathcal{O}_{\mathscr{Y}} \rightarrow \phi_{*} \mathcal{O}_{\mathscr{X}}$ is an isomorphism, and

- ( $\phi$ is cohomologically affine $)$ the functor $\phi_{*}: \mathrm{QCoh}\left(\mathcal{O}_{\mathscr{X}}\right) \rightarrow \mathrm{QCoh}\left(\mathcal{O}_{\mathscr{Y}}\right)$ is exact.

If $\phi: \mathscr{X} \rightarrow \mathscr{Y}$ is such a morphism, then any morphism from $\mathscr{X}$ to an algebraic space factors through $\phi$ [Alp09, Theorem 6.6]. (If $\mathscr{Y}$ is an algebraic space, then this is [Alp09, Theorem 6.6]. More generally, since algebraic spaces are sheaves in the smooth topology, this property may be checked smooth locally on $\mathscr{Y}$, and since good moduli space morphisms are stable under base change [Alp09, Proposition 4.7(i)], this follows from the case of $\mathscr{Y}$ an algebraic space.) In particular, if there exists a good moduli space morphism $\phi: \mathscr{X} \rightarrow X$, where $X$ is an algebraic space, then $X$ is determined up to unique isomorphism. In this case, $X$ is said to be the good moduli space of $\mathscr{X}$. If $\mathscr{X}=[U / G]$, this corresponds to $X$ being a good quotient of $U$ by $G$ in the sense of [GIT94] (for example, for a linearly reductive $G,[\operatorname{Spec} R / G] \rightarrow \operatorname{Spec} R^{G}$ is a good moduli space).

In many respects, good moduli space morphisms behave like proper morphisms. They are universally closed [Alp09, Theorem 4.16(ii)] and weakly separated [ASvdW10, Proposition 2.17], but since points of $\mathscr{X}$ can have non-proper stabilizer groups, good moduli space morphisms are generally not separated (for example, if $G$ is a non-proper group scheme, $B G$ is not separated). Pushforward along a good moduli space morphism respects coherence [Alp09, Theorem 4.16(x)].

Received 19 March 2013, accepted in final form 5 October 2014.

2010 Mathematics Subject Classification 14A20 (primary), 14L15, 14L24, 14L30 (secondary)

Keywords: algebraic stacks, moduli space

This journal is (C) Foundation Compositio Mathematica 2015. This article is distributed with Open Access under the terms of the Creative Commons Attribution Non-Commercial License, which permits non-commercial reuse, distribution, and reproduction in any medium, provided that the original work is properly cited. For commercial re-use, please contact the Foundation Compositio Mathematica.

The second author was partially supported by a National Defense Science and Engineering Graduate Fellowship and by a National Security Agency Young Investigator grant. 


\section{Formal GAGA FOR GOOD MODULI SPACES}

The main theorem in this paper continues this philosophy, showing that formal GAGA holds for good moduli space morphisms, at least when the stack has "enough vector bundles." Recall that a stack is said to have the resolution property if every coherent sheaf has a surjection from a vector bundle. Recall also that if $\mathscr{X} \rightarrow X$ is a good moduli space morphism and $X$ has a unique closed point, then $\mathscr{X}$ also has a unique closed point [Alp09, Theorem 4.16(iii) and Proposition 9.1].

Theorem 1.1. Suppose that $\mathscr{X} \rightarrow \operatorname{Spec} A$ is a good moduli space, where $A$ is a complete Noetherian local ring with maximal ideal $\mathfrak{m}$ and $\mathscr{X}$ is of finite type over $\operatorname{Spec} A$. Let $\widehat{\mathscr{X}}$ denote the formal completion of $\mathscr{X}$ with respect to $\mathfrak{m}$ (see Section 2).

i. The completion functor $\operatorname{Coh}(\mathscr{X}) \rightarrow \operatorname{Coh}(\widehat{\mathscr{X}})$ is fully faithful.

ii. Suppose that $\mathscr{X}_{0}=\mathscr{X} \times_{\operatorname{Spec} A} \operatorname{Spec} A / \mathfrak{m}$ has the resolution property (for example, $\mathscr{X}_{0}$ is a quotient stack; see Remark 3.9). Then the following conditions are equivalent:

(quot) The stack $\mathscr{X}$ is the quotient of an affine scheme by $G L_{n}$ for some $n$.

(quot' $^{\prime}$ ) The stack $\mathscr{X}$ is the quotient of an algebraic space by an affine algebraic group.

These conditions imply the following equivalent conditions:

(res) The stack $\mathscr{X}$ has the resolution property.

$\left(\right.$ res $\left.^{\prime}\right)$ Every coherent sheaf on $\mathscr{X}_{0}$ has a surjection from a vector bundle on $\mathscr{X}$.

These conditions imply the following:

(GAGA) The completion functor $\operatorname{Coh}(\mathscr{X}) \rightarrow \operatorname{Coh}(\widehat{\mathscr{X}})$ is an equivalence.

If the unique closed point of $\mathscr{X}$ has affine stabilizer group, then (res) implies (quot'), and if $\mathscr{X}$ has affine diagonal, then (GAGA) implies $\left(\right.$ res $\left.^{\prime}\right)$.

We provide examples in Section 5 to show that (GAGA) may fail under weaker hypotheses.

Remark 1.2. As this paper went to press, we learned of a forthcoming result by Jarod Alper, Jack Hall, and David Rydh that implies that many stacks satisfy (quot) after an étale base change on their good moduli spaces. Combined with our Theorems 1.1 and 4.4, it implies that if $\mathscr{X}$ has affine diagonal, all the conditions in Theorem 1.1 hold (see Remark 5.7 and Conjecture 5.8).

Remark 1.3. In [Ols05, Theorem 1.4] (see also [Con]), Martin Olsson proves that formal GAGA holds for proper Artin stacks. His main theorem gives a proper surjection from a proper scheme $X \rightarrow \mathscr{X}$, and formal GAGA follows from a dévissage (as outlined in [HR14, § 1.2]). In our setting such a surjection does not exist, and our arguments are quite different.

Remark 1.4. If $\mathscr{X}$ has quasi-finite diagonal over a base $S$, the Hilbert stack $\underline{H}_{\mathscr{X}} \mathscr{X} / S$ of quasi-finite representable $S$-maps with domain a proper $S$-stack is an algebraic stack [HR14, Theorem 2]. A key ingredient in the proof of this result is a weaker variant of formal GAGA for non-separated stacks.

Remark 1.5. Formal GAGA allows the study of a stack $\mathscr{X}$ with good moduli space $X$ to be largely reduced to the study of the fibers of the map $\mathscr{X} \rightarrow X$. This reduction is particularly appealing since it is possible that the geometric fibers of this map must be quotient stacks (see Question 6.4 and Remark 6.7). Here is the template for the reduction:

(0) Start with a problem which is étale local on $X$, and a solution to the problem for the fiber over a point $x$. 


\section{Anton Geraschenko and David Zureick-Brown}

(i) Use deformation theory to extend the solution to a formal solution. Deformation theory typically shows that the problem of extending a solution from an infinitesimal neighborhood to a larger infinitesimal neighborhood is controlled by the cohomologies of certain quasicoherent sheaves. If $\mathscr{X} \rightarrow \operatorname{Spec} A$ has cohomological dimension zero - that is, all higher cohomology groups vanish (which holds for instance if it is cohomologically affine with affine diagonal; see Remark 3.7) - then deformation-theoretic problems are more or less trivial when working with good moduli space morphisms. (See Lemma 3.6 as an example of this.)

(ii) Show that any formal solution is effectivizable. That is, show that any compatible family of solutions over all infinitesimal neighborhoods of $x \in X$ is induced by a solution over Spec $\widehat{\mathcal{O}}_{X, x}$. If the question can be formulated entirely in terms of coherent sheaves, as is often the case, then (GAGA) does this step.

(iii) Use Artin approximation ([Art69, Theorem 1.12]) to extend the solution to an étale neighborhood of $x$. If the stack of solutions is locally finitely presented, Artin's theorem says that for a map $f$ from the complete local ring at a point, there is a map from the henselization of the local ring which agrees with $f$ modulo any given power of the maximal ideal. (By [LO09, Proposition 2.3.8], one can instead apply Artin's theorem to the associated functor of isomorphism classes.) By step (i) (uniqueness of deformations) and formal GAGA, this must actually be an extension of $f$. By local finite presentation, this map extends to some étale neighborhood, as the henselization is the limit of all étale neighborhoods.

Proposition 6.1 illustrates this template. It shows that if $\mathscr{X} \rightarrow X$ is a good moduli space, $x \in X$ is a point at which formal GAGA holds, and the fiber over $x$ is a quotient stack, then there is some étale neighborhood of $x$ over which $\mathscr{X}$ is a quotient stack.

Remark 1.6 (Related work). Previous work [AB05, Theorem 1.7], [AB04, Theorem 7.6], and [Bri13, Theorem 2.20] proves that the Hilbert-scheme of $G$-equivariant multiplicity-finite subschemes of an affine scheme exists when $G$ is connected reductive and of characteristic zero. It follows that formal GAGA holds in the following situation. Working over the spectrum $X$ of a complete local ring, let $V \rightarrow X$ be an affine morphism with an action of a linearly reductive group $G$. Then formal GAGA holds for flat closed substacks $\mathcal{Z}$ of $\mathscr{X}=[V / G]$ such that the good moduli space $Z$ of $\mathcal{Z}$ is finite over $X$. The characteristic $p$ case (with linearly reductive instead of reductive) follows similarly from the existence of the multigraded Hilbert scheme [HS04], since there are few linearly reductive group schemes in characteristic $p$; any such scheme is the extension of a linear reductive finite flat group scheme $G$ by a torus, and [AOV08] classified all such $G$.

The present work is a natural and direct proof of formal GAGA, extending this previous work to non-flat substacks and to arbitrary coherent sheaves. While we work with the more restrictive hypothesis that $Z=X$, David Rydh has pointed out that it is easy to modify our argument to allow for stacks with separated good moduli space of finite type over $X$ and closed substacks $\mathcal{Z}$ whose good moduli space $Z$ is proper over $X$ (and similarly for coherent sheaves). Finally, while our work allows for more general stacks, in the main interesting case where $\mathscr{X}$ has affine stabilizers, our main theorem gives $(\mathrm{GAGA}) \Leftrightarrow($ res $) \Leftrightarrow \mathscr{X}=[V / G]$ with $V$ affine and $G=\mathrm{GL}_{n}$.

\section{Terminology}

This paper follows the conventions of [Alp09]. In particular, all schemes are assumed to be quasi-separated, stacks have quasi-compact diagonal, all morphisms of stacks are assumed to be 


\section{Formal GAGA FOR GOOD MODULI SPACES}

quasi-compact and quasi-separated. We fix a base scheme $X$, which will often be isomorphic to $\operatorname{Spec} A$ where $A$ is a complete Noetherian local ring with maximal ideal $\mathfrak{m}$. An affine algebraic group is understood to be a flat (over $X$ ) subgroup scheme of $\mathrm{GL}_{n}:=\mathrm{GL}_{n, X}$, and a quotient stack $[U / G]$ is the quotient of an algebraic space $U \rightarrow X$ by an affine algebraic group over $S$; in particular, $B G$ always denotes the quotient $[X / G]$ as a stack over $X$.

Throughout the paper $\mathscr{X}$ is an algebraic stack over $X$; unless otherwise indicated, the map $\mathscr{X} \rightarrow X=\operatorname{Spec} A$ is a good moduli space morphism and is of finite type. We denote by $\mathscr{X}_{\text {lis-et }}$ the lisse-étale topos of $\mathscr{X}$ and define $\widehat{\mathcal{O}}_{\mathscr{X}}$ to be the completion $\lim \mathcal{O}_{\mathscr{X}} / \mathcal{I}^{n}$, where $\mathcal{I}$ is the sheaf of ideals generated by the pullback of $\mathfrak{m} \subseteq A$. Following [Con, $\S 1$, we define the ringed topos $\widehat{\mathscr{X}}$ to be the pair $\left(\mathscr{X}_{\text {lis-et }}, \widehat{\mathcal{O}}_{\mathscr{X}}\right)$. There is a natural completion functor

$$
\operatorname{Coh}(\mathscr{X}) \rightarrow \operatorname{Coh}(\widehat{\mathscr{X}}), \quad \mathcal{F} \mapsto \widehat{\mathcal{F}}:=\lim _{\longleftarrow} \mathcal{F} / \mathcal{I}^{n+1} \mathcal{F} .
$$

Letting $\mathscr{X}_{n}=\mathscr{X} \times_{\operatorname{Spec} A} \operatorname{Spec} A / \mathfrak{m}^{n+1}$, the natural functor $\operatorname{Coh}(\widehat{\mathscr{X}}) \rightarrow \lim \operatorname{Coh}\left(\mathscr{X}_{n}\right)$ is an equivalence of categories [Con, Theorem 2.3], where the map $\operatorname{Coh}\left(\mathscr{X}_{n}\right) \rightarrow \widehat{\operatorname{Coh}}\left(\mathscr{X}_{n-1}\right)$ is given by pullback along the closed immersion $\mathscr{X}_{n-1} \rightarrow \mathscr{X}_{n}$. We may therefore regard elements of $\operatorname{Coh}(\widehat{\mathscr{X}})$ as compatible systems of coherent sheaves on the $\mathscr{X}_{n}$.

\section{Proof of Theorem 1.1}

This section is quite technically involved. Subsequent sections depend on the results but not on the techniques or terminology developed in this section.

We use the terminology of topoi developed in [SGA4]. A morphism of topoi $f: Y \rightarrow X$ is a triple $\left(f_{*}, f^{-1}, \alpha\right)$, where $f^{-1}: X \rightarrow Y$ is a functor which commutes with finite limits, $f_{*}: Y \rightarrow X$ is a functor, and $\alpha$ is an adjunction $\operatorname{Hom}_{Y}\left(f^{-1}(-),-\right) \stackrel{\sim}{\rightarrow} \operatorname{Hom}_{X}\left(-, f_{*}(-)\right)$. If $\mathcal{O}_{Y}$ and $\mathcal{O}_{X}$ are sheaves of rings on $Y$ and $X$, respectively, then a morphism of ringed topoi (also denoted $f: Y \rightarrow X)$ is a morphism of topoi, together with a morphism of sheaves of rings $f^{-1} \mathcal{O}_{X} \rightarrow \mathcal{O}_{Y}$. In this case, $f_{*}: \mathcal{O}_{Y}-\bmod \rightarrow \mathcal{O}_{X}-\bmod$ has left adjoint $f^{*}(-)=f^{-1}(-) \otimes_{f^{-1}} \mathcal{O}_{X} \mathcal{O}_{Y}$.

Definition 3.1. A morphism of ringed topoi $f: Y \rightarrow X$ is flat if $f^{*}$ is exact.

Lemma 3.2. If $f: Y \rightarrow X$ is a flat morphism of ringed topoi, $\mathcal{F}$ is a locally finitely presented $\mathcal{O}_{X^{-}}$ module, and $\mathcal{G}$ is any $\mathcal{O}_{X}$-module, then the natural map $f^{*} \mathscr{H}_{\text {om }} \mathcal{O}_{X}(\mathcal{F}, \mathcal{G}) \rightarrow \mathscr{H}$ m $_{\mathcal{O}_{Y}}\left(f^{*} \mathcal{F}, f^{*} \mathcal{G}\right)$ is an isomorphism.

Proof. Case 1: If $\mathcal{F} \cong \mathcal{O}_{X}$, the natural map is isomorphic to the identity map on $f^{*} \mathcal{G}$. Similarly, if $\mathcal{F} \cong \overline{\mathcal{O}_{X}^{\oplus I}}$, the map is isomorphic to the canonical isomorphism $f^{*}\left(\mathcal{G}^{\oplus I}\right) \rightarrow\left(f^{*} \mathcal{G}\right)^{\oplus I}$.

Case 2: Suppose that $\mathcal{F}$ has a global presentation

$$
\mathcal{O}_{X}^{\oplus J} \rightarrow \mathcal{O}_{X}^{\oplus I} \rightarrow \mathcal{F} \rightarrow 0
$$

Since $f^{*}$ is right exact, we get a global presentation

$$
\mathcal{O}_{Y}^{\oplus J} \rightarrow \mathcal{O}_{Y}^{\oplus I} \rightarrow f^{*} \mathcal{F} \rightarrow 0 .
$$

Applying $\mathscr{H} m_{\mathcal{O}_{X}}(-, \mathcal{G})$ to the first sequence and $\mathscr{H}_{0} m_{\mathcal{O}_{Y}}\left(-, f^{*} \mathcal{G}\right)$ to the second, we get the exact sequences

$$
\begin{aligned}
& 0 \longrightarrow \mathscr{H o m}_{\mathcal{O}_{X}}(\mathcal{F}, \mathcal{G}) \longrightarrow \mathscr{H o m}_{\mathcal{O}_{X}}\left(\mathcal{O}_{X}^{\oplus I}, \mathcal{G}\right) \longrightarrow \mathscr{H o m}_{\mathcal{O}_{X}}\left(\mathcal{O}_{X}^{\oplus J}, \mathcal{G}\right) \\
& 0 \rightarrow \mathscr{H o m}_{\mathcal{O}_{Y}}\left(f^{*} \mathcal{F}, f^{*} \mathcal{G}\right) \rightarrow \mathscr{H o m}_{\mathcal{O}_{Y}}\left(\mathcal{O}_{Y}^{\oplus I}, f^{*} \mathcal{G}\right) \rightarrow \mathscr{H o m}_{\mathcal{O}_{Y}}\left(\mathcal{O}_{Y}^{\oplus J}, f^{*} \mathcal{G}\right)
\end{aligned}
$$




\section{Anton Geraschenko and David Zureick-Brown}

Since $f$ is flat, the first sequence remains exact if we apply $f^{*}$, so the rows in the following diagram are exact. The squares commute by naturality of the vertical arrows.

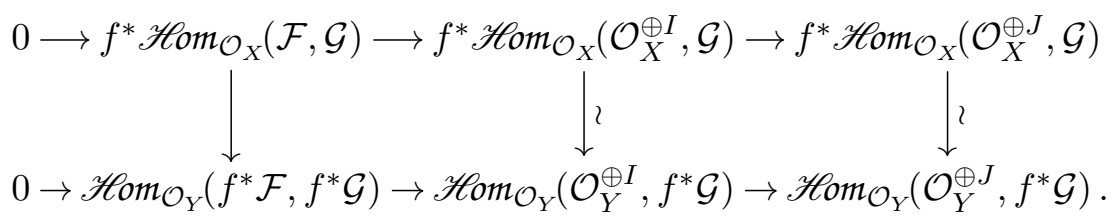

We have already shown that the middle and right vertical arrows are isomorphisms, so the left vertical arrow must also be an isomorphism, completing the proof in the case where $\mathcal{F}$ is globally presented.

Case 3: Now we prove the general case. To check that the natural map $f^{*} \mathscr{H}_{0} m_{\mathcal{O}_{X}}(\mathcal{F}, \mathcal{G}) \rightarrow$ $\mathscr{H}^{\circ} m_{\mathcal{O}_{Y}}\left(f^{*} \mathcal{F}, f^{*} \mathcal{G}\right)$ is an isomorphism, it is enough to find a cover of the final object of $Y$ such that it pulls back to an isomorphism. Since $\mathcal{F}$ is quasi-coherent, there is a cover of the final object of $X$ so that the pullback of $\mathcal{F}$ has a presentation. Pulling that cover back along $f$, we get a cover of the final object of $Y$ (here we are using the exactness of $f^{-1}$ to say that the final object pulls back to the final object and that covers pull back to covers on canonical sites). On that cover, the map is an isomorphism by case 2 . The construction of $\mathscr{H}$ om, the application of $f^{*}$, and the construction of the natural map are local on $X$, so the natural morphism constructed on the cover is the restriction of the natural morphism on $Y$.

Lemma 3.3. If $\mathscr{X}$ is a Noetherian algebraic stack and $\mathcal{I} \subseteq \mathcal{O}_{\mathscr{X}}$ is a quasi-coherent sheaf of ideals, then $\widehat{\mathcal{O}}_{\mathscr{X}}$, the completion of $\mathcal{O}_{\mathscr{X}}$ with respect to $\mathcal{I}$, is flat over $\mathcal{O}_{\mathscr{X}}$. That is, the canonical map $\iota: \widehat{\mathscr{X}} \rightarrow \mathscr{X}$ is a flat morphism of ringed topoi.

Proof. Let $\mathcal{F} \rightarrow \mathcal{F}^{\prime}$ be an injection of $\mathcal{O}_{\mathscr{X}}$-modules. We need to check the injectivity of the map

$$
\mathcal{F} \otimes \mathcal{O}_{\mathscr{X}} \widehat{\mathcal{O}}_{\mathscr{X}} \rightarrow \mathcal{F}^{\prime} \otimes_{\mathcal{O}_{\mathscr{X}}} \widehat{\mathcal{O}}_{\mathscr{X}}
$$

Since sheafification is exact, it suffices to check the injectivity of the maps

$$
\mathcal{F}(U) \otimes_{\mathcal{O}_{\mathscr{X}}(U)} \widehat{\mathcal{O}}_{\mathscr{X}}(U) \rightarrow \mathcal{F}^{\prime}(U) \otimes_{\mathcal{O}_{\mathscr{K}}(U)} \widehat{\mathcal{O}}_{\mathscr{X}}(U)
$$

as $U$ varies over a base for $\mathscr{X}_{\text {lis-et }}$. Thus it suffices to check that the maps above are injections for $f: U \rightarrow \mathscr{X}$ a smooth map and $U$ an affine scheme. By definition, $\mathcal{O}_{\mathscr{X}}(U)=\mathcal{O}_{U}(U)$ and $\widehat{\mathcal{O}}_{\mathscr{X}}(U)=\widehat{\mathcal{O}}_{U}(U)$. Since $U$ is affine, $\left.\widehat{\mathcal{O}}_{U}(U)=\widehat{\mathcal{O}_{U}(U}\right)$. Injectivity follows since $\widehat{\mathcal{O}_{U}(U)}$ is flat over $\mathcal{O}_{U}(U)$ [Eis95, Theorem 7.2b].

Remark 3.4. The same trick of restricting to affine schemes smooth over $\mathscr{X}$ shows that for any coherent sheaf $\mathcal{F}$ on $\mathscr{X}$, the natural map $\iota^{*} \mathcal{F} \rightarrow \widehat{\mathcal{F}}$ is an isomorphism. (Note, however, that this is not true for quasi-coherent sheaves.)

Remark 3.5. Lemma 3.3 and Remark 3.4 show that completion of coherent sheaves is exact.

Lemma 3.6. Suppose that $\phi: \mathscr{X} \rightarrow \operatorname{Spec} A$ is a good moduli space, where $A$ is a complete Noetherian local ring with maximal ideal $\mathfrak{m}$. Additionally assume that $\phi$ has cohomological dimension zero. Then any vector bundle $\mathcal{V}$ on $\mathscr{X}_{n-1}$ is the reduction of a unique vector bundle on $\mathscr{X}_{n}$. In particular, any vector bundle on $\mathscr{X}_{0}$ extends to a unique vector bundle on $\widehat{\mathscr{X}}$.

Remark 3.7 (Cohomological dimension of cohomologically affine morphisms). If $\mathscr{X}$ has affine diagonal, then $\phi$ has cohomological dimension zero; that is, $R^{i} \phi_{*}=0$ for $i>0$. Indeed, by [Alp09, 


\section{Formal GAGA FOR GOOD MODULI SPACES}

Remark 3.5]), cohomologically affine stacks with non-affine diagonal are not cohomologically of dimension zero. The reason is that the morphism of triangulated categories

$$
D^{+}(\mathrm{QCoh}(X)) \rightarrow D_{\mathrm{QCoh}}^{+}\left(\mathcal{O}_{X} \text {-mod }\right)
$$

is not an isomorphism unless $\mathscr{X}$ has affine diagonal, and the derived functors are computed in the second category. (See for example [SP, Tag 07B5].)

An easy example is that for an elliptic curve $E$ over a field $k, f:$ Spec $k \rightarrow B E$ is not cohomologically of dimension zero; this follows from pulling back by the smooth cover $f: \operatorname{Spec} k \rightarrow B E$ and cohomology and base change. A more essential counterexample is the structure morphism $g: B E \rightarrow$ Spec $k$, the hypercover spectral sequence associated with $f:$ Spec $k \rightarrow B E$ gives $\mathrm{H}^{1}\left(B E, \mathcal{O}_{B E}\right) \neq 0$.

Proof of Lemma 3.6. This is a direct application of [FGI ${ }^{+}$05, Theorem 8.5.3(b)]. The obstruction to extending $\mathcal{V}$ to $\mathscr{X}_{n}$ lies in $\mathrm{H}^{2}\left(\mathscr{X}_{n-1}, \mathcal{I}^{n} \otimes \mathcal{E} n d(\mathcal{V})\right)$, which vanishes since $\mathscr{X}_{n-1}$ is cohomologically of dimension zero. Therefore $\mathcal{V}$ extends. Moreover, the isomorphism classes of extensions are parameterized by $\mathrm{H}^{1}\left(\mathscr{X}_{n-1}, \mathcal{I}^{n} \otimes \mathcal{E} n d(\mathcal{V})\right)$, which vanishes by the same argument, so the extension is unique.

Lemma 3.8. Suppose that $\phi: \mathscr{X} \rightarrow \operatorname{Spec} A$ is a good moduli space, where $A$ is a complete Noetherian local ring with maximal ideal $\mathfrak{m}$. Then a quasi-coherent sheaf $\mathcal{F}$ on a locally Noetherian stack $\mathscr{X}$ is a flat $\mathcal{O}_{\mathscr{X}}$-module (that is, restricts to a flat sheaf on any smooth cover by a scheme) if and only if $\mathcal{F} \otimes_{\mathcal{O}_{\mathscr{X}}}-$ is an exact functor on $\mathrm{QCoh}(\mathscr{X})$.

Proof. Suppose that $\mathcal{F}$ is flat and let $\mathcal{G} \rightarrow \mathcal{G}^{\prime}$ be an injection of quasi-coherent sheaves. Let $f: U \rightarrow \mathscr{X}$ be a smooth cover by a scheme. We may check that $\mathcal{F} \otimes \mathcal{G} \rightarrow \mathcal{F} \otimes \mathcal{G}^{\prime}$ is injective after pulling back to $U$. Pullback respects tensor products, $f^{*} \mathcal{G} \rightarrow f^{*} \mathcal{G}^{\prime}$ is injective (since $f$ is flat), and $f^{*} \mathcal{F}$ is a flat $\mathcal{O}_{U}$-module, so $f^{*}(\mathcal{F} \otimes \mathcal{G}) \rightarrow f^{*}\left(\mathcal{F} \otimes \mathcal{G}^{\prime}\right)$ is injective.

For the converse, again let $f: U \rightarrow \mathscr{X}$ be a smooth cover by a scheme. We wish to prove that $f^{*} \mathcal{F}$ is flat. This may be done locally on $U$, so we may assume that $U$ is a Noetherian affine scheme. The result is well known for schemes, so it suffices to prove that $f^{*} \mathcal{F} \otimes_{\mathcal{O}_{U}}-$ is an exact functor on $\mathrm{QCoh}(U)$. First we claim that for any $\mathcal{O}_{U}$-module $\mathcal{G}$, the counit of adjunction $f^{*} f_{*} \mathcal{G} \rightarrow \mathcal{G}$ has a natural section. Indeed, let $W \rightarrow U$ be a smooth morphism. Then the map

$$
f^{*} f_{*} \mathcal{G}(W \rightarrow U) \cong \mathcal{G}(U \times \mathscr{X} W \rightarrow U) \rightarrow \mathcal{G}(W \rightarrow U)
$$

has a section given by the restriction map

$$
\mathcal{G}(W \rightarrow U) \rightarrow \mathcal{G}(U \times \mathscr{X} W \rightarrow W \rightarrow U) .
$$

Now let $\mathcal{G} \rightarrow \mathcal{G}^{\prime}$ be an injection of quasi-coherent sheaves on $U$. Since $U$ is Noetherian, $f$ is quasi-compact and quasi-separated, so $f_{*} \mathcal{G} \rightarrow f_{*} \mathcal{G}^{\prime}$ is an injection of quasi-coherent $\mathcal{O}_{\mathscr{X}}$-modules. By assumption, $\mathcal{F} \otimes_{\mathcal{O}_{\mathscr{X}}} f_{*} \mathcal{G} \rightarrow \mathcal{F} \otimes_{\mathcal{O}_{\mathscr{X}}} f_{*} \mathcal{G}^{\prime}$ is an injection, and since $f$ is flat, $\phi: f^{*}\left(\mathcal{F} \otimes_{\mathcal{O}_{\mathscr{X}}} f_{*} \mathcal{G}\right) \rightarrow$ $f^{*}\left(\mathcal{F} \otimes_{\mathcal{O}_{\mathscr{X}}} f_{*} \mathcal{G}^{\prime}\right)$ is an injection. Noting that $f^{*}\left(\mathcal{F} \otimes_{\mathcal{O}_{\mathscr{X}}} f_{*}(-)\right) \cong f^{*} \mathcal{F} \otimes_{\mathcal{O}_{U}} f^{*} f_{*}(-)$, we get a diagram

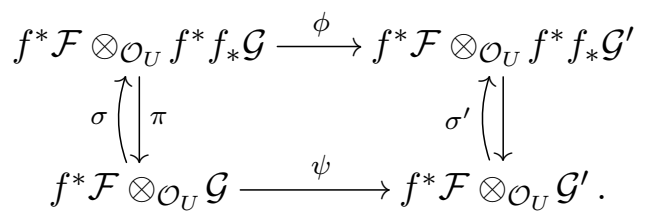

We have that $\phi$ is injective, and $\sigma$ is injective (since it is a section of $\pi$ ). Since $\sigma^{\prime} \circ \psi=\phi \circ \sigma$, we conclude that $\psi$ is injective. 


\section{Anton Geraschenko and David Zureick-Brown}

Proof of Theorem 1.1. Part (i): For any coherent $\mathcal{O}_{\mathscr{X}}$-modules $\mathcal{F}$ and $\mathcal{G}$, we must show that the natural map $\operatorname{Hom}_{\mathcal{O}_{\mathscr{X}}(\mathcal{F}, \mathcal{G})} \rightarrow \operatorname{Hom}_{\widehat{\mathcal{O}} \mathscr{X}}(\widehat{\mathcal{F}}, \widehat{G})$ is an isomorphism. We have that $\mathscr{H}$ om $(\mathcal{F}, \mathcal{G})$ is coherent. By Lemma 3.2, Lemma 3.3, and Remark 3.4, the natural map $\left.\widehat{\mathscr{H}_{0} \text { O }_{\mathscr{X}}(\mathcal{F}}, \mathcal{G}\right) \rightarrow$ $\mathscr{H}^{\circ} m_{\widehat{\mathcal{O}}}(\widehat{\mathcal{F}}, \widehat{\mathcal{G}})$ is an isomorphism. By [Alp09, Proposition 4.7 (iii)], the induced map on global sections is the desired isomorphism.

Part (ii): (res) $\Rightarrow\left(\right.$ res $\left.^{\prime}\right)$. This is immediate since any coherent sheaf on $\mathscr{X}_{0}$ is a coherent sheaf on $\mathscr{X}$.

$\left(\right.$ res $\left.^{\prime}\right) \Rightarrow($ GAGA). By part (i), the completion functor is fully faithful. It remains to show that any compatible system $\mathfrak{F}=\left\{\mathcal{F}_{n}\right\}_{n} \geqslant 0$ of coherent sheaves on the stacks $\mathscr{X}_{n}$ is induced by a coherent sheaf $\mathcal{F}$ on $\mathscr{X}$. As usual, we denote by $\mathcal{I}$ the quasi-coherent sheaf of ideals generated by $\phi^{*}(\mathfrak{m})$.

By $\left(\right.$ res $\left.^{\prime}\right)$, there exist a locally free sheaf $\mathcal{V}$ on $\mathscr{X}$ and a surjection $\mathcal{V} \rightarrow \mathcal{F}_{0}$. We inductively argue that for each $n$ this lifts to a surjection $\mathcal{V} \rightarrow \mathcal{F}_{n}$. The bottom row of the following diagram is exact:

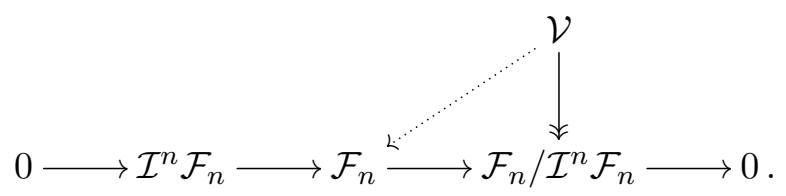

Since $\mathcal{V}$ is a vector bundle, the following sequence is exact:

$$
0 \rightarrow \mathscr{H} m_{\mathcal{O}_{\mathscr{X}}}\left(\mathcal{V}, \mathcal{I}^{n} \mathcal{F}_{n}\right) \rightarrow \mathscr{H} m_{\mathcal{O}_{\mathscr{X}}}\left(\mathcal{V}, \mathcal{F}_{n}\right) \rightarrow \mathscr{H} m_{\mathcal{O}_{\mathscr{X}}}\left(\mathcal{V}, \mathcal{F}_{n} / \mathcal{I}^{n} \mathcal{F}_{n}\right) \rightarrow 0
$$

By cohomological affineness of $\phi$, the sequence remains exact when we take global sections, so the composition map $\operatorname{Hom}_{\mathcal{O}}\left(\mathcal{V}, \mathcal{F}_{n}\right) \rightarrow \operatorname{Hom}_{\mathcal{O}}\left(\mathcal{V}, \mathcal{F}_{n} / \mathcal{I}^{n} \mathcal{F}_{n}\right)$ is surjective. Thus, there is a lift $\mathcal{V} \rightarrow \mathcal{F}_{n}$ as indicated by the dotted arrow in (1). The induced map $\mathcal{V} \rightarrow \mathcal{F}_{n}$ is surjective by Nakayama's lemma. This gives a compatible system of maps $\left\{\mathcal{V} \rightarrow \mathcal{F}_{m}\right\}_{m \geqslant 0}$, and thus a surjective morphism $\widehat{\mathcal{V}} \rightarrow \mathfrak{F}$.

Repeating the argument above for the kernel of $\widehat{\mathcal{V}} \rightarrow \mathfrak{F}$, we get a presentation $\widehat{\mathcal{W}} \rightarrow \widehat{\mathcal{V}} \rightarrow \mathfrak{F} \rightarrow$ 0 , where $\mathcal{V}$ and $\mathcal{W}$ are vector bundles on $\mathscr{X}$. By part (i), the morphism $\widehat{\mathcal{W}} \rightarrow \widehat{\mathcal{V}}$ is induced by some $\mathcal{O}_{\mathscr{X}}$-module homomorphism $\mathcal{W} \rightarrow \mathcal{V}$. Let $\mathcal{G}$ be the cokernel of this map. By Remark 3.5, the top row of the following diagram is exact:

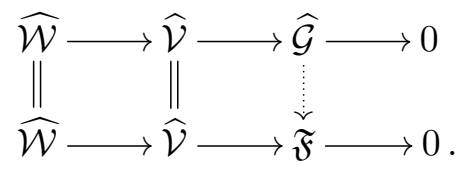

The induced morphism from $\widehat{\mathcal{G}}$ to $\mathfrak{F}$ is therefore an isomorphism.

$\left(\right.$ res $\left.^{\prime}\right) \Rightarrow($ res $)$. The argument above shows that if $\left(\right.$ res $\left.^{\prime}\right)$ holds and $\mathcal{F}$ is a coherent sheaf on $\mathscr{X}$, then there are a vector bundle $\mathcal{V}$ on $\mathscr{X}$ and a surjection $\widehat{\mathcal{V}} \rightarrow \widehat{\mathcal{F}}$. Since $\left(\right.$ res $\left.^{\prime}\right) \Rightarrow($ GAGA), this map is induced by a surjection $\mathcal{V} \rightarrow \mathcal{F}$.

$($ GAGA $) \Rightarrow\left(\right.$ res $\left.^{\prime}\right)$. First we show that if $\mathscr{X}$ has affine diagonal and if (GAGA) holds, any $\mathcal{F} \in$ $\operatorname{Coh}(\mathscr{X})$ whose completion is a vector bundle on $\widehat{\mathscr{X}}$ is a vector bundle. By Remark 3.4 , the equivalence of categories of coherent sheaves respects tensor products, so since $\widehat{\mathcal{F}} \otimes_{\widehat{\mathcal{O}}_{\mathscr{X}}}$ - is an exact functor on $\operatorname{Coh}(\widehat{\mathscr{X}})$, we have that $\mathcal{F} \otimes_{\mathcal{O}_{\mathscr{X}}}-$ is an exact functor on $\operatorname{Coh}(\mathscr{X})$. Let $\operatorname{Spec} R \rightarrow \mathscr{X}$ be a smooth cover of $\mathscr{X}$ (note that $\mathscr{X}$ is assumed of finite type over the Noetherian ring $A$, so it is quasi-compact). Then $\operatorname{Spec} R \times \mathscr{X} \operatorname{Spec} R$ is of finite type over $\operatorname{Spec} A$, so the projections 


\section{Formal GAGA FOR GOOD MODULI SPACES}

$\operatorname{Spec} R \times \mathscr{X}$ Spec $R \rightarrow \operatorname{Spec} R$ are smooth, quasi-compact, and quasi-separated, so any quasicoherent sheaf on $\mathscr{X}$ is the limit of its coherent subsheaves [SP, Lemma 07TU]. Since $\mathcal{F} \otimes_{\mathcal{O}_{\mathscr{X}}}-$ commutes with direct limits, it is exact on the category of quasi-coherent sheaves, so $\mathcal{F}$ is a flat $\mathcal{O}_{\mathscr{X}}$-module by Lemma 3.8. It follows that $\mathcal{F}$ is a vector bundle; indeed, this can be checked smooth locally, and a flat coherent sheaf on a Noetherian affine scheme is locally free [Mil80, Theorem 2.9 of Chapter 1].

Now for any coherent sheaf $\mathcal{F}_{0}$ on $\mathscr{X}_{0}$, since $\mathscr{X}_{0}$ is assumed to have the resolution property, there is a vector bundle $\mathcal{V}_{0}$ on $\mathscr{X}_{0}$ with a surjection to $\mathcal{F}_{0}$. By Lemma 3.6, $\mathcal{V}_{0}$ extends to a vector bundle on $\widehat{\mathscr{X}}$, which by formal GAGA is the completion of a coherent sheaf $\mathcal{V}$ on $\mathscr{X}$. By the paragraph above, $\mathcal{V}$ is a vector bundle. Now $\mathcal{V} \rightarrow \mathcal{V}_{0} \rightarrow \mathcal{F}_{0}$ is a surjection. This shows that (res') holds.

(quot) $\Leftrightarrow\left(\right.$ quot $\left.^{\prime}\right)$. It is clear that $($ quot $) \Rightarrow\left(\right.$ quot $\left.^{\prime}\right)$. Conversely, suppose $\mathscr{X}=[V / G]$ for some algebraic space $V$ and some subgroup $G \subseteq \mathrm{GL}_{n}$. Let $U=\left(V \times \mathrm{GL}_{n}\right) / G$, where $g \cdot(v, h)=$ $\left(v \cdot g^{-1}, g \cdot h\right)$ (alternatively, $U$ is the pullback of the universal $\mathrm{GL}_{n}$-torsor along the composition $\left.[V / G] \rightarrow B G \rightarrow B \mathrm{GL}_{n}\right)$. Then $\mathscr{X}=\left[U / \mathrm{GL}_{n}\right]$.

Since $U \rightarrow \mathscr{X}$ is a $\mathrm{GL}_{n}$-torsor, it is an affine morphism, and $\mathscr{X} \rightarrow \operatorname{Spec} A$ is cohomologically affine, so $U \rightarrow \operatorname{Spec} A$ is cohomologically affine. As $U$ has trivial stabilizers, it is an algebraic space, so by Serre's criterion [Knu71, Theorem III.2.5], $U$ is an affine scheme.

(quot) $\Rightarrow($ res $) \Rightarrow\left(\right.$ quot $\left.^{\prime}\right)$. By [Gro13, Corollary 5.9] (quot) $\Rightarrow($ res), and if the closed point of $\mathscr{X}$ has affine stabilizer, then by [Tot04, Lemma 4.1] (res) $\Rightarrow\left(\right.$ quot $\left.^{\prime}\right)$.

Remark 3.9. The proofs of (quot) $\Leftrightarrow\left(\right.$ quot $\left.^{\prime}\right)$ and (quot) $\Rightarrow($ res $) \Rightarrow\left(\right.$ quot $\left.^{\prime}\right)$ apply to any stack with affine good moduli space. Note however that $(\mathrm{res}) \Rightarrow\left(\right.$ quot $\left.^{\prime}\right)$ requires all closed points of the stack to have affine stabilizer.

Remark 3.10. Note that the proof of $(\mathrm{GAGA}) \Rightarrow\left(\mathrm{res}^{\prime}\right)$ shows that (GAGA) implies that any coherent sheaf whose completion is a vector bundle must be a vector bundle. The hypothesis that $\mathscr{X}_{0}$ have the resolution property is not necessary for this result.

Remark 3.11. Suppose $A / \mathfrak{m}=k$ and that $A$ is a $k$-algebra (this is automatic if $k$ has characteristic zero $^{1}$ ). If $\mathscr{X} \cong \mathscr{X}_{0} \times_{\operatorname{Spec} k} \operatorname{Spec} A$, then we have a morphism $s: \mathscr{X} \rightarrow \mathscr{X}_{0}$ such that $\mathscr{X}_{0} \hookrightarrow \mathscr{X} \stackrel{s}{\rightarrow}$ $\mathscr{X}_{0}$ is the identity map. Any vector bundle $\mathcal{V}_{0} \in \operatorname{Coh}\left(\mathscr{X}_{0}\right)$ is the reduction of the vector bundle $s^{*} \mathcal{V}_{0} \in \operatorname{Coh}(\mathscr{X})$. If $\mathscr{X}_{0}$ has the resolution property, then any $\mathcal{F}_{0} \in \operatorname{Coh}\left(\mathscr{X}_{0}\right)$ has a surjection from a vector bundle $\mathcal{V}_{0} \in \operatorname{Coh}\left(\mathscr{X}_{0}\right)$, so the map $s^{*} \mathcal{V}_{0} \rightarrow \mathcal{V}_{0} \rightarrow \mathcal{F}_{0}$ is a surjection from a vector bundle on $\mathscr{X}$. That is, if $\mathscr{X}_{0}$ has the resolution property, (res') holds.

Note, however, that the condition $\mathscr{X} \cong \mathscr{X}_{0} \times_{\operatorname{Spec} k} \operatorname{Spec} A$ is frequently not satisfied. For example, consider the $j$-invariant map $j: \mathcal{M}_{1,1} \rightarrow \mathbb{A}_{\mathbb{C}}^{1}$ and let $\mathscr{X} \rightarrow$ Spec $\mathbb{C} \llbracket t \rrbracket$ be the pullback of $j$ to the local ring of $\mathbb{A}_{\mathbb{C}}^{1}$ at the origin. Since elliptic curves with $j$-invariant zero have automorphism group $\mathbb{Z} / 4 \mathbb{Z}$ but generic elliptic curves have automorphism group $\mathbb{Z} / 2 \mathbb{Z}, \mathscr{X}$ cannot be the pullback of its special fiber.

\section{Formal GAGA is finite flat local (and étale local) on the base}

Lemma 4.1. Suppose that $\phi: \mathscr{X} \rightarrow \operatorname{Spec} A$ is a good moduli space, where $A$ is a complete Noetherian local ring and $\phi$ is of finite type. Suppose that $\operatorname{Spec} A^{\prime} \rightarrow \operatorname{Spec} A$ is a finite flat

\footnotetext{
${ }^{1}$ Every non-negative integer is non-zero in $k$, so lies in $A \backslash \mathfrak{m}$, so is invertible in $A$. This shows that $A$ is a $\mathbb{Q}$-algebra. By [Eis95, Theorem 7.7], it is a $k$-algebra.
} 


\section{Anton Geraschenko and David Zureick-Brown}

morphism, where $A^{\prime} \neq 0$ is again local (and therefore a complete Noetherian local ring), and let $\mathscr{X}^{\prime}=\mathscr{X} \times \operatorname{Spec} A \operatorname{Spec} A^{\prime}$.

If $\operatorname{Coh}\left(\mathscr{X}^{\prime}\right) \rightarrow \operatorname{Coh}\left(\widehat{\mathscr{X}}^{\prime}\right)$ is essentially surjective, then so is $\operatorname{Coh}(\mathscr{X}) \rightarrow \operatorname{Coh}(\widehat{\mathscr{X}})$.

Remark 4.2. We note that any morphism of spectra of complete Noetherian local rings Spec $A^{\prime} \rightarrow$ $\operatorname{Spec} A$ which is an étale cover must be finite flat. Since such a morphism is surjective, $A^{\prime} / \mathfrak{m}_{A} A^{\prime}$ is some étale (and so finite) extension of $A / \mathfrak{m}_{A}$. In particular, $\mathfrak{m}_{A} A^{\prime}=\mathfrak{m}_{A^{\prime}}$. By [EGA IV, Proposition 18.3.2], there is a finite étale morphism $\operatorname{Spec} B \rightarrow \operatorname{Spec} A$ inducing the same extension of $A / \mathfrak{m}_{A}$. By the formal criterion for étaleness and the fact that $A^{\prime}$ and $B$ are each complete with respect to $\mathfrak{m}_{A}$, there are unique morphisms $\operatorname{Spec} A^{\prime} \rightarrow \operatorname{Spec} B$ and $\operatorname{Spec} B \rightarrow \operatorname{Spec} A^{\prime}$ over Spec $A$ lifting the isomorphism of extensions of $A / \mathfrak{m}_{A}$, and these must be inverses. Thus, $\operatorname{Spec} A^{\prime} \rightarrow \operatorname{Spec} A$ is finite flat.

Remark 4.3. By Remark 3.4, completion of coherent sheaves agrees with pullback along the morphism of topoi $\iota: \widehat{\mathscr{X}} \rightarrow \mathscr{X}$. It follows that pullback along $\pi: \mathscr{X}^{\prime} \rightarrow \mathscr{X}$ commutes with completion of coherent sheaves, and that completion of coherent sheaves is a right-exact functor. To see this, note that the hypotheses of $A^{\prime} \neq 0$ and finiteness imply that $\mathfrak{m}_{A^{\prime}}^{n} \subseteq \mathfrak{m}_{A} A^{\prime} \subseteq \mathfrak{m}_{A^{\prime}}$ for some $n$. Indeed, the second containment can only fail if $\mathfrak{m}_{A}$ contains a unit of $A^{\prime}$, in which case $\mathfrak{m}_{A} A^{\prime}=A^{\prime}$, so Nakayama's lemma implies $A^{\prime}=0$. For the first containment, we can reduce to the case $\mathfrak{m}_{A}=0$, so $A$ is a field. Then $A^{\prime}$ is a finite-dimensional vector space, so $\mathfrak{m}_{A^{\prime}}^{n}$ stabilizes for large $n$, and it must stabilize to 0 (again by Nakayama). We can therefore regard both $\widehat{\mathscr{X}}$ and $\widehat{\mathscr{X}}^{\prime}$ as completions with respect to the pullback of $\mathfrak{m}_{A}$.

Proof of Lemma 4.1. Good moduli space morphisms are stable under base change [Alp09, Proposition 4.7(i)] and composition, so $\mathscr{X}^{\prime \prime}=\mathscr{X}^{\prime} \times \mathscr{X} \mathscr{X}^{\prime} \rightarrow \operatorname{Spec} A^{\prime \prime}=\operatorname{Spec}\left(A^{\prime} \otimes_{A} A^{\prime}\right)$ is a $\operatorname{good}$ moduli space. Let $p_{1}, p_{2}: \mathscr{X}^{\prime \prime} \rightarrow \mathscr{X}^{\prime}$ denote the projections. While $A^{\prime \prime}$ may no longer be a local ring, $\operatorname{Spec} A^{\prime \prime}$ is finite flat over $\operatorname{Spec} A^{\prime}$, so it must be a disjoint union $\bigsqcup \operatorname{Spec} A_{i}^{\prime \prime}$, where each $A_{i}^{\prime \prime}$ is a complete local ring. Let $\mathscr{X}_{i}^{\prime \prime}=\mathscr{X}^{\prime \prime} \times_{\operatorname{Spec}} A^{\prime \prime} \operatorname{Spec} A_{i}^{\prime \prime}$.

Let $\mathfrak{F} \in \operatorname{Coh}(\widehat{\mathscr{X}})$, and let $\mathfrak{F}^{\prime} \in \operatorname{Coh}\left(\widehat{\mathscr{X}}^{\prime}\right)$ be the pullback to $\widehat{\mathscr{X}}^{\prime}$. By assumption, $\mathfrak{F}^{\prime}$ is the completion of a sheaf $\mathcal{F}^{\prime} \in \operatorname{Coh}\left(\mathscr{X}^{\prime}\right)$. Applying Theorem 1.1(i) to each of the good moduli space morphisms $\mathscr{X}_{i}^{\prime \prime} \rightarrow \operatorname{Spec} A_{i}^{\prime \prime}$, we see that the descent datum $p_{2}^{*} \mathfrak{F}^{\prime} \stackrel{\sim}{\rightarrow} p_{1}^{*} \mathfrak{F}^{\prime}$ is induced by a map $p_{2}^{*} \mathcal{F}^{\prime} \stackrel{\sim}{\rightarrow} p_{2}^{*} \mathcal{F}^{\prime}$ (note that we are using Remark 4.3). By faithfully flat of finite presentation (fppf) descent for coherent sheaves, $\mathcal{F}^{\prime}$ is the pullback of a coherent sheaf $\mathcal{F}$ on $\mathscr{X}$. Since $\widehat{\mathcal{F}}$ and $\mathfrak{F}$ are defined by the same descent datum, they are isomorphic.

ThEOREM 4.4 (Formal GAGA is finite flat and étale local on the base). In the setup of Lemma 4.1, formal GAGA holds for $\mathscr{X} \rightarrow \operatorname{Spec} A$ if and only if it holds for $\mathscr{X}^{\prime} \rightarrow \operatorname{Spec} A^{\prime}$.

Proof. By Theorem 1.1(i), both completion functors are fully faithful.

By Lemma 4.1 , if the completion functor $\operatorname{Coh}\left(\mathscr{X}^{\prime}\right) \rightarrow \operatorname{Coh}\left(\widehat{\mathscr{X}}^{\prime}\right)$ is essentially surjective, then so is $\operatorname{Coh}(\mathscr{X}) \rightarrow \operatorname{Coh}(\widehat{\mathscr{X}})$.

Conversely, suppose that $\operatorname{Coh}(\mathscr{X}) \rightarrow \operatorname{Coh}(\widehat{\mathscr{X}})$ is essentially surjective, and let $\mathfrak{F} \in \operatorname{Coh}\left(\widehat{\mathscr{X}}^{\prime}\right)$. Since $\pi: \mathscr{X}^{\prime} \rightarrow \mathscr{X}$ is finite, $\pi_{*} \mathfrak{F} \in \operatorname{Coh}(\widehat{\mathscr{X}})$. By assumption, $\pi_{*} \mathfrak{F} \cong \widehat{\mathcal{F}}$ for some $\mathcal{F} \in \operatorname{Coh}(\mathscr{X})$. The composition $\pi^{*} \widehat{\mathcal{F}} \stackrel{\sim}{\rightarrow} \pi^{*} \pi_{*} \mathfrak{F} \rightarrow \mathfrak{F}$ is a surjection. Let $\mathfrak{G}$ denote the kernel of this map. By the same argument, there exists a surjection $\pi^{*} \widehat{\mathcal{G}} \rightarrow \mathfrak{G}$ for some $\mathcal{G} \in \operatorname{Coh}(\mathscr{X})$. Then $\mathfrak{F}$ is the cokernel of the map $\pi^{*} \widehat{\mathcal{G}} \rightarrow \pi^{*} \widehat{\mathcal{F}}$. By full faithfulness and Remark 4.3, this map is induced by a morphism $\pi^{*} \mathcal{G} \rightarrow \pi^{*} \mathcal{F}$, and the cokernel of this map has completion $\mathfrak{F}$.

Remark 4.5. In the same spirit, we note that the resolution property also descends along finite flat morphisms [Gro13, Proposition 4.3 (vii)]. 


\section{Formal GAGA FOR GOOD MODULI SPACES}

\section{Counterexamples to formal GAGA}

Recall that for a relative group scheme $G \rightarrow S$, a coherent sheaf on $B G=[S / G]$ is equivalent to a coherent sheaf on $S$ with a $G$-linearization (that is, a $G$-action). Pushforward along $\phi: B G \rightarrow S$ corresponds to taking the subsheaf of invariants; in particular, since $\mathcal{O}_{B G}$ corresponds to $\mathcal{O}_{S}$ with the trivial $G$-action, $\phi$ is Stein. Since the action of $G$ on $S$ is trivial, $\phi$ is universal for maps to algebraic spaces. ${ }^{2}$ The condition that the map be cohomologically affine is precisely the condition that $G$ be linearly reductive. Therefore $B G \rightarrow S$ is a good moduli space if and only if $G$ is linearly reductive. (We note that this holds even if $G$ is not affine; while in the usual definition of reductive of [SGA3] $G \rightarrow S$ is affine, we use the notion of linearly reductive of [Alp09, Definition 12.1], which is a cohomological condition.)

Formal GAGA fails without the good moduli space condition. In the following, we say that a morphism to an algebraic space $\mathscr{X} \rightarrow X$ is a no-good moduli space if it is universal for maps to algebraic spaces but is not a good moduli space.

Example 5.1 (Counterexample to full faithfulness for a no-good moduli space). Let $A=k \llbracket t \rrbracket$ for a field $k$ of characteristic not two. Let $G=\operatorname{Spec} k \llbracket t \rrbracket \sqcup \operatorname{Spec} k((t))$, regarded as an open subgroup of $(\mathbb{Z} / 2 \mathbb{Z})_{\operatorname{Spec} A}$. Then $\mathscr{X}=B G \rightarrow \operatorname{Spec} A$ is not a good moduli space. The non-trivial onedimensional representation of $\mathbb{Z} / 2 \mathbb{Z}$ induces a non-trivial rank one vector bundle on $\mathscr{X}$ whose completion is the trivial rank one vector bundle on $\widehat{\mathscr{X}}$ (indeed, $\widehat{\mathscr{X}} \cong \operatorname{Spec} A$ ), showing that the completion functor is not fully faithful.

Example 5.2 (Counterexample to essential surjectivity for a no-good moduli space). Formal GAGA fails for $B \mathbb{G}_{a}$. For a ring $R$, a line bundle on $B \mathbb{G}_{a, R}$ is equivalent to a one-dimensional representation of $\mathbb{G}_{a, R}$ (that is, a group homomorphism $\mathbb{G}_{a, R} \rightarrow \mathbb{G}_{m, R}$ ). The formula $x \mapsto \exp (t x)=$ $\sum_{i=0}^{\infty} \frac{t^{i}}{i !} x^{i}$ gives a compatible family of homomorphisms $\mathbb{G}_{a, \mathbb{C}[t] / t^{n}} \rightarrow \mathbb{G}_{m, \mathbb{C}[t] / t^{n}}$ which do not lift to a homomorphism $\mathbb{G}_{a, \mathbb{C} \llbracket t \rrbracket} \rightarrow \mathbb{G}_{m, \mathbb{C} \llbracket t \rrbracket}$.

Formal GAGA may also fail for good moduli spaces.

Example 5.3 (Counterexample to essential surjectivity with non-separated diagonal). Let $A=$ $k \llbracket t \rrbracket$ for a field $k$. Let $G$ be Spec $k \llbracket t \rrbracket$ with a doubled origin, regarded as a group over $\operatorname{Spec} A$. Since $G$ is a quotient of $(\mathbb{Z} / 2 \mathbb{Z})_{\operatorname{Spec} A}$ by a flat subgroup scheme, it is linearly reductive by [Alp09, Proposition 12.17], so $\mathscr{X}=B G \rightarrow \operatorname{Spec} A$ is a good moduli space.

Any vector bundle on $\mathscr{X}$ consists of a vector bundle $\mathcal{V}$ on Spec $A$ and a group homomorphism $G_{A} \rightarrow \operatorname{Aut}_{A}(\mathcal{V})$. Since $\operatorname{Aut}_{A}(\mathcal{V})$ is separated, such a map must factor through the trivial group. So any vector bundle on $\mathscr{X}$ corresponds to a vector bundle on $\operatorname{Spec} A$ with trivial $G$-action. However, $\widehat{\mathscr{X}} \cong B_{\operatorname{Spec}} A(\mathbb{Z} / 2 \mathbb{Z})$, so there are formal vector bundles not of this form, namely those induced by non-trivial representations of $\mathbb{Z} / 2 \mathbb{Z}$.

Even if we require separated diagonal, formal GAGA may still fail.

Example 5.4 (Counterexample to essential surjectivity with separated, non-affine diagonal). Let

$$
G^{\prime}=\operatorname{Proj}\left(k \llbracket t \rrbracket[x, y, z] /\left(z y^{2}-x^{2}(x+z)-t z^{3}\right)\right),
$$

where $t$ has degree zero and $x, y$, and $z$ have degree one. Let $G$ be the complement of the origin of the special fiber, with structure map $\pi: G \rightarrow$ Spec $k \llbracket t \rrbracket$. The generic fiber is an elliptic curve

${ }^{2}$ More generally, if $\alpha: G \times X \rightarrow X$ is an action of $G$ on an algebraic space $X$ and the two maps $\alpha, p_{2}: G \times X \rightarrow X$ 


\section{Anton Geraschenko and David Zureick-Brown}

$E \rightarrow$ Spec $k((t))$, but the special fiber is isomorphic to $\mathbb{G}_{m}$. By [Sil94, IV Theorem 5.3(c)], $G$ is a relative group scheme over Spec $k \llbracket t \rrbracket$. We claim that $B G \rightarrow \operatorname{Spec} k \llbracket t \rrbracket$ is a good moduli space morphism (that is, that taking $G$-invariants is exact on $G$-linearized coherent sheaves).

To see this, we first note that any deformation of the group scheme $\mathbb{G}_{m}$ is trivial. By [SGA3, Exposé III, Corollaire 3.9], isomorphism classes of deformations of the group scheme along a square-zero ideal $I$ (if they exist) are parameterized by $\mathrm{H}^{2}\left(\mathbb{G}_{m}, \operatorname{Lie}\left(\mathbb{G}_{m}\right) \otimes I\right)$, where $\operatorname{Lie}\left(\mathbb{G}_{m}\right)$ is the adjoint representation and $I$ has the trivial action. The group cohomology $\mathrm{H}^{i}\left(\mathbb{G}_{m},-\right)$ as defined in [SGA3, Exposé III, 1.1] is simply the Čech cohomology associated with the cover Spec $k \llbracket t \rrbracket \rightarrow B \mathbb{G}_{m}$. Since $\mathbb{G}_{m}$ is affine, this Cech cohomology agrees with sheaf cohomology on $B \mathbb{G}_{m}$. Since $\mathbb{G}_{m}$ is linearly reductive, $B \mathbb{G}_{m} \rightarrow$ Spec $k \llbracket t \rrbracket$ is cohomologically affine, so the higher cohomology groups vanish. Thus, the only deformation of $\mathbb{G}_{m}$ is $\mathbb{G}_{m}$.

Next, any torsion $G$-linearized coherent sheaf is supported over $\operatorname{Spec}\left(k[t] / t^{n}\right)$ for some $n$. That is, there is some choice of $n$ such that the given sheaf is in the essential image of $j_{*}$ in the following diagram:

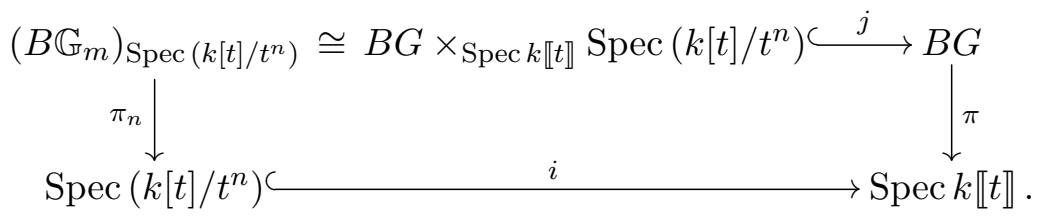

Since $i$ and $j$ are affine, and $\pi_{n}$ is cohomologically affine, we have

$$
R \pi_{*} \circ j_{*}=R\left(\pi_{*} \circ j_{*}\right)=R\left(i_{*} \circ \pi_{n *}\right)=i_{*} \circ \pi_{n *}=\pi_{*} \circ j_{*} .
$$

That is, torsion sheaves on $B G$ have trivial higher cohomology.

Any torsion-free $G$-linearized coherent sheaf is free with trivial action. Indeed, it is free with some rank $r$ since $k \llbracket t \rrbracket$ is a discrete valuation ring The action of $G$ is given by some group homomorphism $G \rightarrow \mathrm{GL}_{r, k \llbracket t \rrbracket}$. Since $G$ has proper connected generic fiber and $\mathrm{GL}_{r}$ is affine, this map must be trivial over the generic point. Since $G$ is reduced and $\mathrm{GL}_{r}$ is separated, the map must be trivial.

Any $G$-linearized coherent sheaf $\mathcal{F}$ (with torsion subsheaf $\mathcal{F}^{\text {tor }}$ ) fits into a $G$-equivariant short exact sequence

$$
0 \rightarrow \mathcal{F}^{\text {tor }} \rightarrow \mathcal{F} \rightarrow \mathcal{F} / \mathcal{F}^{\text {tor }} \rightarrow 0 \text {. }
$$

Since $\mathcal{F} / \mathcal{F}^{\text {tor }}$ is free, the following sequence is exact.

$$
0 \rightarrow \mathscr{H o m}\left(\mathcal{F} / \mathcal{F}^{\text {tor }}, \mathcal{F}^{\text {tor }}\right) \rightarrow \mathscr{H o m}\left(\mathcal{F} / \mathcal{F}^{\text {tor }}, \mathcal{F}\right) \rightarrow \mathscr{H o m}\left(\mathcal{F} / \mathcal{F}^{\text {tor }}, \mathcal{F} / \mathcal{F}^{\text {tor }}\right) \rightarrow 0
$$

Since $\mathscr{H}$ om $\left(\mathcal{F} / \mathcal{F}^{\text {tor }}, \mathcal{F}^{\text {tor }}\right)$ is torsion, $\mathrm{H}^{1}\left(B G, \mathscr{H} \operatorname{lom}\left(\mathcal{F} / \mathcal{F}^{\text {tor }}, \mathcal{F}^{\text {tor }}\right)\right)=0$, so the sequence remains exact when we take global sections. Global sections of $\mathscr{H}$ om $(\mathcal{F}, \mathcal{G})$ are $G$-equivariant maps from $\mathcal{F}$ to $\mathcal{G}$, so there is a $G$-equivariant splitting of the sequence (2). We have shown that any $G$ linearized coherent $k \llbracket t \rrbracket$-module $M$ decomposes into a direct sum of its torsion part $M^{\text {tor }}$ (with

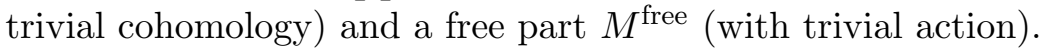

Suppose that we have a short exact sequence of linearized modules $0 \rightarrow M^{\prime \prime} \rightarrow M \stackrel{\phi}{\rightarrow} M^{\prime} \rightarrow 0$. We wish to show that any invariant $m^{\prime} \in M^{\prime}$ is the image of an invariant element of $M$. Since $\phi$ is surjective, we have $m^{\prime}=\phi\left(m_{f}+m_{t}\right)$, where $m_{t}$ is torsion and $m_{f}$ is invariant. Since torsion sheaves have trivial cohomology, any invariant torsion element which is the image of a torsion element is actually the image of an invariant torsion element, so $\phi\left(m_{t}\right)=\phi\left(n_{t}\right)$ for some invariant torsion element $n_{t} \in M$. Then $m_{f}+n_{t}$ is invariant and $\phi\left(m_{f}+n_{t}\right)=m^{\prime}$. This completes the 


\section{FORMAL GAGA FOR GOOD MODULI SPACES}

proof that $B G \rightarrow$ Spec $k \llbracket t \rrbracket$ is a good moduli space morphism.

Now take any vector bundle over the origin with non-trivial $\mathbb{G}_{m}$ action. By Lemma 3.6, this extends to a unique vector bundle on $\widehat{B G}$, but we have seen that there is no torsion-free coherent sheaf on $B G$ with non-trivial action on the special fiber.

Remark 5.5. A similar example gives a counterexample to [Alp10, Conjecture 1]. Let

$$
G^{\prime}=\operatorname{Proj}\left(\mathbb{C}[t, x, y, z] /\left(z y^{2}-x^{2}(x+z)-t z^{3}\right)\right),
$$

where $t$ has degree zero and $x, y$, and $z$ have degree one. Let $G$ be the largest subscheme of $G^{\prime}$ over which the map to $\mathbb{A}^{1}=\operatorname{Spec} \mathbb{C}[t]$ is smooth. By [Sil94, IV Theorem 5.3(c)], $G$ is a relative group over $\mathbb{A}^{1}$. Let $\mathscr{X}=B G$. This $\mathscr{X}$ is finitely presented over Spec $\mathbb{C}$, and the image under the quotient map $\mathbb{A}^{1} \rightarrow \mathscr{X}$ of the origin of $\mathbb{A}^{1}$ is a closed point $x$ with stabilizer $\mathbb{G}_{m}$. If [Alp10, Conjecture 1] were true, there would be an algebraic space $Y$ with a point $y$ and an étale representable morphism $f:\left[Y / \mathbb{G}_{m}\right] \rightarrow \mathscr{X}$ sending $y$ to $x$, inducing an isomorphism of stabilizers. As $f$ is étale, its image is open, so the image contains some closed point of $\mathscr{X}$ whose stabilizer is an elliptic curve. By [GS15, Proposition 3.2], $f$ induces finite-index inclusions of stabilizers. But no subgroup of $\mathbb{G}_{m}$ can possibly be a finite-index subgroup of an elliptic curve.

It is possible that [Alp10, Conjecture 1] holds for stacks with affine stabilizers.

Remark 5.6. Taking $\mathscr{X}=B G$ and $\mathscr{X}^{\prime}=B \mathbb{G}_{m}$ over $A=k \llbracket t \rrbracket$, Example 5.4 shows that the natural map

$$
\operatorname{Hom}_{A}\left(\mathscr{X}, \mathscr{X}^{\prime}\right) \rightarrow \operatorname{Hom}_{A}\left(\widehat{\mathscr{X}}, \widehat{\mathscr{X}}^{\prime}\right)
$$

is not necessarily an equivalence of categories. The complex analytic analogue of this natural map is an equivalence of categories if $\mathscr{X}$ is a proper Deligne-Mumford stack and $\mathscr{X}^{\prime}$ is either a quasi-compact algebraic stack with affine diagonal [Lur04, Theorem 1.1] or a locally of finite type Deligne-Mumford stack with quasi-compact and quasi-separated diagonal [Hal14, Theorem 1].

Remark 5.7. It is difficult to imagine an example of a stack $\mathscr{X}$ with affine diagonal and good moduli space $\operatorname{Spec} A$ which is not a quotient stack (that is, does not satisfy (quot')) étale locally on Spec $A$. Likely candidates, such as non-trivial $\mathbb{G}_{m}$-gerbes, do not work (see Remark 6.5). If no such stack exists, then Theorems 1.1 and 4.4 show that formal GAGA holds provided that $\mathscr{X}$ has affine diagonal.

Conjecture 5.8. Suppose that $\phi: \mathscr{X} \rightarrow \operatorname{Spec} A$ is a good moduli space morphism, where $A$ is a complete Noetherian local ring and $\phi$ is of finite type. If $\mathscr{X}$ has affine diagonal, then the completion functor $\operatorname{Coh}(\mathscr{X}) \rightarrow \operatorname{Coh}(\widehat{\mathscr{X}})$ is an equivalence of categories. (Note: a forthcoming result implies that this conjecture holds; see Remark 1.2.)

Formal GAGA may hold even if $\mathscr{X}$ does not have affine diagonal, but it is usually uninteresting. For example, for any elliptic curve $E \rightarrow \operatorname{Spec} A$, formal GAGA holds for $B E \rightarrow \operatorname{Spec} A$ since all coherent sheaves on $B E$ are pulled back from Spec $A$.

\section{Application to the local quotient structure of good moduli spaces}

Recall that a stack $\mathscr{X}$ is a quotient stack if it is the stack quotient of an algebraic space by a subgroup of $\mathrm{GL}_{n}$ for some $n$ (that is, if (quot') holds).

Proposition 6.1. Let $\phi: \mathscr{X} \rightarrow X$ be a stack over $X$ with affine diagonal, with $\phi$ of finite type and $X$ a locally Noetherian scheme. Assume that $\phi$ is a good moduli space. Let $x \in X$ be a 


\section{Anton Geraschenko and David Zureick-Brown}

point such that the fiber $\mathscr{X}_{0}$ over $x$ is a quotient stack. Suppose that formal GAGA holds for $\widetilde{\mathscr{X}}=\mathscr{X} \times_{X} \operatorname{Spec} \widehat{\mathcal{O}}_{X, x} \rightarrow \operatorname{Spec} \widehat{\mathcal{O}}_{X, x}$. Then there exists an étale neighborhood $X^{\prime} \rightarrow X$ of $x$ such that $\mathscr{X} \times_{X} X^{\prime}$ is a quotient stack.

Remark 6.2. To apply the proposition to the case where $X$ is an algebraic space and $x$ is a topological point, one would find an étale neighborhood $U \rightarrow X$ with $U$ a scheme and a point $u \in U$ which maps to $x$, then apply the proposition to $\mathscr{X} \times{ }_{X} U \rightarrow U$. Any two étale covers have a common refinement, so by Theorem 4.4, the formal GAGA hypothesis is satisfied for one étale cover if and only if it is satisfied for any étale cover. (Also see Remark 6.3.)

Proof of Proposition 6.1. The question is étale local on $X$, and by Theorem 4.4 the hypothesis is étale local on $X$, so we may assume that $X=\operatorname{Spec} R$ is an affine scheme. Let $X^{h}=\operatorname{Spec} R^{h}$, where $R^{h}$ is the strict henselization of $R$ at $x$, and let $X^{l o c}=\operatorname{Spec} \widehat{R^{h}}$. Let $\mathscr{X}^{l o c}$ and $\mathscr{X}^{h}$ denote the pullback of $\mathscr{X}$ to $X^{l o c}$ and $X^{h}$, respectively. For a sheaf $\mathcal{F}$ on $\mathscr{X}$ (or $\mathscr{X}^{h}$ ), let $\mathcal{F}^{h}$ and $\mathcal{F}^{\text {loc }}$ denote the pullback of $\mathcal{F}$ to $\mathscr{X}^{h}$ and $\mathscr{X}^{l o c}$, respectively. Let $\widehat{\mathscr{X}}$ be the completion of $\mathscr{X}^{l o c}$ with respect to the maximal ideal of $\widehat{R^{h}}$. We have the following commutative diagram:

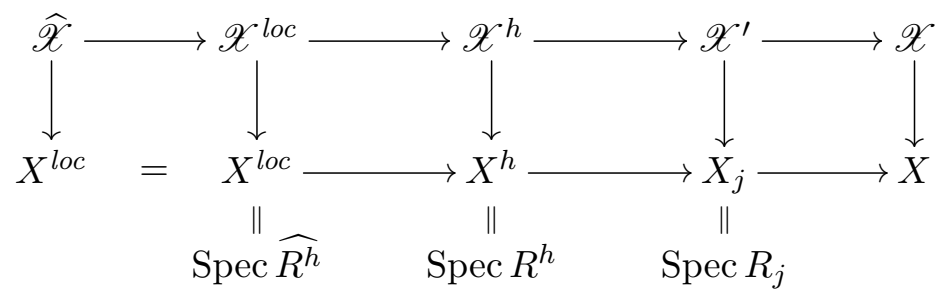

The closed substack $\mathscr{X}_{0} \subseteq \widetilde{\mathscr{X}}$ is a quotient stack, so its unique closed point has affine stabilizer, and it has the resolution property by Remark 3.9. By assumption, (GAGA) holds for $\widetilde{\mathscr{X}} \rightarrow$ Spec $\widehat{\mathcal{O}}_{X, x}$, so by Theorem 1.1(ii), $\widetilde{\mathscr{X}}=\left[U / \mathrm{GL}_{n}\right]$ for an affine scheme $U$. Since Spec $\widehat{R^{h}} \rightarrow$ Spec $\widehat{\mathcal{O}}_{X, x}$ is an affine morphism, $\mathscr{X}^{l o c} \rightarrow \widetilde{\mathscr{X}}$ is affine, so $U^{l o c}=U \times \widetilde{\mathscr{X}}^{\text {Xoc }}$ is an affine scheme and $\mathscr{X}^{l o c}=\left[U^{l o c} / \mathrm{GL}_{n}\right]$. By Remark 3.9, $\mathscr{X}^{l o c}$ has the resolution property.

Next, we show that $\mathscr{X}^{h}$ has the resolution property. Let $\mathcal{F}$ be a coherent sheaf on $\mathscr{X}^{h}$. By the previous paragraph, there is a vector bundle $\mathcal{V}^{l o c}$ on $\mathscr{X}^{l o c}$ with a surjection to $\mathcal{F}^{l o c}$. By [LMB00, Proposition 4.18(i)], the stack of rank $n$ vector bundles on $\mathscr{X}, \mathscr{H}$ om $\left(\mathscr{X}, B \mathrm{GL}_{n}\right)$, is locally of finite presentation over $X$. By Artin approximation [Art69, Theorem 1.12], there exists a vector bundle $\mathcal{V}$ on $\mathscr{X}^{h}$ such that the pullback of $\mathcal{V}$ to $\mathscr{X}_{0}$ is the same as the pullback of $\mathcal{V}^{l o c}$ to $\mathscr{X}_{0}$. By Lemma 3.6 and Theorem 1.1(i), the pullback of $\mathcal{V}$ to $\mathscr{X}^{l o c}$ is isomorphic to $\mathcal{V}^{l o c}$. Since $\mathscr{H} \operatorname{mom}(\mathcal{V}, \mathcal{F})$ is locally of finite presentation and the substack of surjections is open in $\mathscr{H}$ om $(\mathcal{V}, \mathcal{F})$ [Lie06, Lemma 2.2.2], the substack of surjections is locally of finite presentation. By Artin approximation, there exists a surjection $\mathcal{V} \rightarrow \mathcal{F}$. This proves that $\mathscr{X}^{h}$ has the resolution property.

Let $\mathscr{X}_{0}^{h}$ denote the closed fiber of $\mathscr{X}^{h}$. The morphism $\mathscr{X}_{0}^{h} \rightarrow \mathscr{X}_{0}$ is a representable morphism to a quotient stack. If $\mathscr{X}_{0}=[U / G]$, then $\mathscr{X}_{0}^{h}=\left[\left(U \times \mathscr{X}_{0} \mathscr{X}_{0}^{h}\right) / G\right]$, so $\mathscr{X}_{0}^{h}$ is a quotient stack. In particular, the closed point of $\mathscr{X}^{h}$ has affine stabilizer, so by Remark $3.9, \mathscr{X}^{h}=\left[P^{h} / \mathrm{GL}_{n}\right]$ for some affine scheme $P^{h}$. The $\mathrm{GL}_{n}$-torsor $P^{h} \rightarrow \mathscr{X}^{h}$ corresponds to a representable map $p^{h}: \mathscr{X}^{h} \rightarrow B \mathrm{GL}_{n}$. Since $\mathscr{H} \operatorname{Com}\left(\mathscr{X}, B \mathrm{GL}_{n}\right)$ is locally of finite presentation over $X^{h}$ and since $R^{h}=\lim _{\longrightarrow} R_{i}$, where the limit runs over all étale neighborhoods $X_{i}=\operatorname{Spec} R_{i} \rightarrow \operatorname{Spec} R$ of $x$, we have that $p^{h}$ is the pullback of some $p_{i}: \mathscr{X}_{i}=\mathscr{X} \times_{X} X_{i} \rightarrow B \mathrm{GL}_{n}$. Let $\mathcal{Q}_{i} \rightarrow \mathscr{X}_{i}$ be 


\section{Formal GAGA FOR GOOD MODULI SPACES}

the corresponding $\mathrm{GL}_{n}$-torsor. To finish the proof, it suffices to show that there exists an étale neighborhood $X_{j} \rightarrow X_{i}$ such that $\mathcal{Q}_{j}=\mathcal{Q}_{i} \times_{X_{i}} X_{j}$ is an affine scheme.

Since $X$ is locally Noetherian, $X^{h}$ is Noetherian [EGA IV, Proposition 18.8.8(iv)]. As $P^{h}$ is of finite type over $X^{h}$, it is finitely presented over $X^{h}$, so there exist an étale neighborhood $X_{j_{0}} \rightarrow X_{i}$ and an affine scheme $P_{j_{0}}$ over $X_{j_{0}}$ such that we have $P^{h} \cong P_{j_{0}} \times_{X_{j_{0}}} X^{h}$. Let $\mathcal{Q}_{j_{0}}=$ $\mathcal{Q}_{i} \times_{X_{i}} X_{j_{0}}$. By [LMB00, Proposition 4.18(i)], $\mathscr{H}_{0} m_{X_{j_{0}}}\left(\mathcal{Q}_{j_{0}}, P_{j_{0}}\right)$ and $\mathscr{H}_{0} m_{X_{j_{0}}}\left(P_{j_{0}}, \mathcal{Q}_{j_{0}}\right)$ are locally of finite presentation over $X_{j_{0}}$, so there exists an étale neighborhood $X_{j_{1}} \rightarrow X_{j_{0}}$ such that the isomorphism $f: \mathcal{Q}_{i} \times_{X} X^{h}=\mathcal{Q}_{j_{0}} \times_{X_{j_{0}}} X^{h} \rightarrow P_{j_{0}} \times_{X_{j_{0}}} X^{h}=P^{h}$ and its inverse $g$ are the pullbacks of maps $f_{1}$ and $g_{1}$ which are defined over $X_{j_{1}}$. By [LMB00, Proposition 4.18(i)], there is an étale neighborhood $X_{j} \rightarrow X_{j_{1}}$ such that the compositions $f_{1} \circ g_{1}$ and $g_{1} \circ f_{1}$ pull back to the identities over $X_{j}$. This shows that $\mathcal{Q}_{j} \cong P_{j}$ is an affine scheme, as desired.

Remark 6.3. In the proof of Proposition 6.1, the formal GAGA hypothesis is only used to show that $\mathscr{X}^{\text {loc }}$ has the resolution property. If this can be obtained in some other way (for example if formal GAGA holds for $\mathscr{X}^{l o c} \rightarrow \operatorname{Spec} \widehat{\mathcal{O}_{X, x}^{h}}$, the rest of this proof works as above.

Because of results like Proposition 6.1, and more generally because of the strategy presented in Remark 1.5, it is desirable to have a classification of stacks which have a point as a good moduli space. It is not known which such stacks are quotient stacks.

Question 6.4. Does there exist a good moduli space morphism $\mathscr{Y} \rightarrow \operatorname{Spec} k$, with $k$ a separably closed field, such that $\mathscr{Y}$ has affine diagonal but is not a quotient stack? (Note: a forthcoming result resolves this question in the negative; see Remark 1.2.)

Remark 6.5. One natural source of examples is non-trivial gerbes. By [EHKV01, Example 3.12] there are $\mathbb{G}_{m}$-gerbes which are not quotient stacks. If $\mathscr{X}$ is a $\mathbb{G}_{m}$-gerbe over a Noetherian scheme $X$, then $\mathscr{X}$ is a quotient stack if and only if its class in $\mathrm{H}^{2}\left(X, \mathbb{G}_{m}\right)$ is in the image of the Brauer map $\operatorname{Br}(X) \rightarrow \mathrm{H}^{2}\left(X, \mathbb{G}_{m}\right)$ [EHKV01, Theorem 3.6]. However, if $X=\operatorname{Spec} A$, where $A$ is a complete local ring (for example a field), then by [Mil80, Corollary IV.2.12], ${ }^{3}$ the natural map $\operatorname{Br}(X) \rightarrow \mathrm{H}^{2}\left(X, \mathbb{G}_{m}\right)$ is an isomorphism, so any $\mathbb{G}_{m}$-gerbe over Spec $A$ is a quotient stack.

Remark 6.6. Another candidate counterexample is $\mathfrak{M}_{0}^{\leqslant m}$, the moduli stack of genus zero prestable (that is, nodal) curves with at most $m$ nodes. Over any field $K$ and for any $m \geqslant 2, \mathfrak{M}_{0}^{\leqslant m}$ is not a quotient stack [Kre13, Proposition 5.2]. The map $\mathfrak{M}_{0}^{\leqslant m} \rightarrow \operatorname{Spec} K$ is universal for maps to algebraic spaces. However, it is not a good moduli space map since closed points of $\mathfrak{M}_{0}^{\leqslant m}$ can have non-reductive stabilizers: each outer leaf of any tree $T$ of smooth rational curves contributes a copy of Aut $\mathbb{A}^{1} \cong \mathbb{G}_{m} \ltimes \mathbb{G}_{a}$ to AutT. But by [Alp09, Proposition 12.14], the stabilizers at closed points of a stack which has a good moduli space are linearly reductive.

A promising variant is $\mathfrak{M}_{0, n}^{\leqslant m}$, the moduli stack of marked genus zero prestable curves with $n$ marked points and at most $m$ nodes, such that each component has at least two marks/nodes. The closed points of this stack have linearly reductive stabilizers, and the stack is non-empty for $n \geqslant 2$. For $m \geqslant 2$, we sketch a modification of Kresch's argument to show that $\mathfrak{M}_{0, n}^{\leqslant m}$ is not a quotient stack. There is an open immersion $\mathfrak{M}_{0, n}^{\leqslant 2} \subseteq \mathfrak{M}_{0, n}^{\leqslant m}$, so it suffices to show that $\mathfrak{M}_{0, n}^{\leqslant 2}$ is not a quotient stack. There is a representable morphism $\widetilde{\mathfrak{M}}_{0, n}^{\leqslant 2} \rightarrow \mathfrak{M}_{0, n}^{\leqslant 2}$ from the stack in which the points are labeled, so it suffices to check that the former is not a quotient stack. There is a morphism $\widetilde{\mathfrak{M}}_{0,2}^{\leqslant 2} \rightarrow \widetilde{\mathfrak{M}}_{0, n}^{\leqslant 2}$ given by adding points in a prescribed fashion, which is a trivial

\footnotetext{
${ }^{3}$ Note that in contrast to [EHKV01], Milne defines $\operatorname{Br}^{\prime}(X)=\mathrm{H}^{2}\left(X, \mathbb{G}_{m}\right)$ (p. 147).
} 


\section{Anton Geraschenko and David Zureick-Brown}

$\mathbb{G}_{m}$-gerbe over its image (for $n \geqslant 3$ ), so it suffices to check that $\widetilde{\mathfrak{M}}_{0,2}^{\leqslant 2}$ is not a quotient stack. A straightforward modification of the proof of [Kre13, Proposition 5.2] shows that $\widetilde{\mathfrak{M}}_{0,2}^{\leqslant 2}$ is not a quotient stack.

For $n \geqslant 3$ and $m \geqslant 2$, there are curves which isotrivially degenerate to multiple closed points, so $\mathfrak{M}_{0, n}^{\leqslant m}$ cannot have a good moduli space by [Alp09, Proposition 4.16(iii)]. The stack $\mathfrak{M}_{0,2}^{\leqslant 2}$ has a unique closed point (topologically, it is a chain of three points), and the map to a point is universal for maps to algebraic spaces. If this map were a good moduli space morphism, it would answer Question 6.4 affirmatively.

Remark 6.7. Suppose that $\mathscr{X} \rightarrow \operatorname{Spec} A$ is a good moduli space as in Section 2, with $k=A / \mathfrak{m}$ separably closed. Suppose that $\mathscr{X}$ has affine diagonal, and satisfies [Alp10, Conjecture 1] (by Remark 5.5, we cannot expect this unless $\mathscr{X}$ has affine stabilizers). Let $G_{x}$ be the stabilizer of the unique closed point $x$ of $\mathscr{X}$. Then there are a representable étale morphism $f: \mathcal{W}=\left[U / G_{x}\right] \rightarrow \mathscr{X}$ and a point $w \in \mathcal{W}(k)$ such that the induced map $\operatorname{Aut}_{\mathcal{W}(k)}(w) \rightarrow \operatorname{Aut}_{\mathscr{X}(k)}(x)=G_{x}$ is an isomorphism. Suppose that the strong form of this conjecture holds (that is, that we may take $U=\operatorname{Spec} R$ to be affine; see [Alp10, second paragraph after Conjecture 1]).

(This argument was suggested to us by Jarod Alper.) Let $\mathcal{W}=\left[\operatorname{Spec} R / G_{x}\right] \rightarrow \mathscr{X}$ be as above. By [Alp09, Theorem 5.1], the induced map on good moduli spaces $\operatorname{Spec} R^{G_{x}} \rightarrow \operatorname{Spec} A$ is étale. Since $A$ is complete with separably closed residue field, the component of $\operatorname{Spec} R^{G_{x}}$ containing the image of $w$ must be isomorphic to $\operatorname{Spec} A$, so after shrinking $\operatorname{Spec} R$, we may assume that $f: \mathcal{W} \rightarrow \mathscr{X}$ induces an isomorphism of good moduli spaces.

We claim that $f$ is an isomorphism. Since $f$ is étale, its image is open. Any open set containing the unique closed point $x$ of $\mathscr{X}$ is all of $\mathscr{X}$, so $f$ is an étale cover. We may check that a morphism is an isomorphism étale locally on the base, so it suffices to show that the projection $p_{1}: \mathcal{W} \times \mathscr{X} \mathcal{W} \rightarrow \mathcal{W}$ is an isomorphism. By [Alp09, Proposition 4.7(i)] $\mathcal{W} \times \mathscr{X} \mathcal{W}$ has good moduli space $\operatorname{Spec} A \times \operatorname{Spec} A \operatorname{Spec} A=\operatorname{Spec} A$, so it has a unique closed point. The diagonal $\mathcal{W} \rightarrow \mathcal{W} \times \mathscr{X} \mathcal{W}$ has this closed point in its image. As the diagonal is a section of an étale morphism, it is an open immersion, so it is an isomorphism.

The strong form of [Alp10, Conjecture 1] for stacks with affine diagonal therefore answers Question 6.4 negatively: if $A=k$ is a separably closed field, the argument above shows that $\mathscr{X}$ is a quotient stack.

Remark 6.8. If the strong form of [Alp10, Conjecture 1] for stacks with affine diagonal is true, the following argument shows that Conjecture 5.8 is true. In this case, the formal GAGA hypothesis in Proposition 6.1 may be replaced by the hypothesis that $\mathscr{X}$ has affine diagonal.

Let $G_{x}$ denote the stabilizer of the closed point $x$ of $\mathscr{X}$. By [Alp09, Proposition 12.14], $G_{x}$ is linearly reductive. Let $\mathcal{W}=\left[\operatorname{Spec} R / G_{x}\right] \rightarrow \mathscr{X}$ and $w \in \mathcal{W}$ be as in [Alp10, Conjecture 1]. By [Alp09, Theorem 5.1], the map on good moduli spaces $\operatorname{Spec} R^{G_{x}} \rightarrow \operatorname{Spec} A$ is étale, so after shrinking $\operatorname{Spec} R$, we may assume that $\operatorname{Spec} R^{G_{x}} \rightarrow \operatorname{Spec} A$ is a finite étale extension. As $\mathcal{W}$ and $\mathscr{X} \times_{\operatorname{Spec} A} \operatorname{Spec} R^{G_{x}}$ are both étale over $\mathscr{X}$, the induced morphism $\mathcal{W} \rightarrow \mathscr{X} \times \operatorname{Spec} A$ Spec $R^{G_{x}}$ is étale. This morphism induces an isomorphism on good moduli spaces, and the image contains the unique closed point of $\mathscr{X} \times_{\operatorname{Spec} A} \operatorname{Spec} R^{G_{x}}$. By the argument in Remark 6.7, the map is an isomorphism. As $\mathcal{W}$ is a quotient stack, formal GAGA holds for $\mathcal{W} \rightarrow \operatorname{Spec} R^{G_{x}}$ by Theorem 1.1(ii). By Theorem 4.4, formal GAGA holds for $\mathscr{X} \rightarrow \operatorname{Spec} A$. 


\section{FORMAL GAGA FOR GOOD MODULI SPACES}

\section{ACKNOWLEDGEMENTS}

We thank Jarod Alper, who introduced us to the problem and generously provided valuable feedback throughout our work on it. We also thank Jack Hall, Martin Olsson, David Rydh, and Matt Satriano for many helpful discussions. We thank David Ben-Zvi, Bhargav Bhatt, Scott Carnahan, Torsten Ekedahl, David Speyer, Angelo Vistoli, Ben Webster, and Jonathan Wise for useful discussions on MathOverflow. We especially thank the anonymous referee, who provided extensive useful comments, and thank Jack Hall and David Rydh a second time for very detailed comments, and the correspondence about related work, which became Remark 1.6.

\section{REFERENCES}

AB04 V. Alexeev and M. Brion, Stable reductive varieties I: Affine varieties, Invent. Math. 157 (2004), no. 2, 227-274. http://dx.doi.org/10.1007/s00222-003-0347-y

AB05 V. Alexeev and M. Brion, Moduli of affine schemes with reductive group action, J. Algebraic Geom. 14 (2005), no. 1, 83-117. http://dx.doi.org/10.1090/S1056-3911-04-00377-7

Alp09 J. Alper, Good moduli spaces for Artin stacks, Ann. Inst. Fourier (Grenoble) 63 (2013), no. 6, 2349-2402. http://dx.doi.org/10.5802/aif.2833

Alp10 J. Alper, On the local quotient structure of Artin stacks, J. Pure Appl. Algebra 214 (2010), no. 9, 1576-1591. http://dx.doi.org/10.1016/j.jpaa.2009.11.016

AOV08 D. Abramovich, M. Olsson, and A. Vistoli, Tame stacks in positive characteristic, Ann. Inst. Fourier (Grenoble), 58 (2008), no. 4, 1057-1091. http://dx.doi.org/10.5802/ aif. 2378

Art69 M. Artin, Algebraic approximation of structures over complete local rings, Publ. Math. Inst. Hautes Études Sci. 36, 23-58, 1969. http://dx.doi.org/10.1007/BF02684596

ASvdW10 J. Alper, D. Smyth, and F. van der Wyck, Weakly proper moduli stacks of curves, arXiv: 1012.0538

Bri13 M. Brion, Invariant Hilbert schemes in Handbook of moduli, Vol. I, vol. 24, Adv. Lect. Math., 64-117, Int. Press, Somerville, MA, 2013.

Con B. Conrad, Formal GAGA on Artin stacks, available at http://math.stanford.edu/ conrad/papers/formalgaga.pdf.

EGA IV A. Grothendieck, Éléments de géométrie algébrique IV-4, Étude locale des schémas et des morphismes de schémas IV, Publ. Math. Inst. Hautes Études Sci. 32 (1967).

EHKV01 D. Edidin, B. Hassett, A. Kresch, and A. Vistoli, Brauer groups and quotient stacks, Amer. J. Math. 123 (2001), no. 4, 761-777. http://dx.doi.org/10.1353/ajm.2001.0024

Eis95 D. Eisenbud, Commutative algebra, with a view toward algebraic geometry, Grad. Texts in Math., vol. 150, Springer-Verlag, New York, 1995. http://dx.doi.org/10.1007/ 978-1-4612-5350-1

$\mathrm{FGI}^{+} 05$ B. Fantechi, L. Göttsche, L. Illusie, S. L. Kleiman, N. Nitsure, and A. Vistoli, Fundamental algebraic geometry, Grothendieck's FGA explained, Math. Surveys Monogr., vol. 123, Amer. Math. Soc., Providence, RI, 2005. http://dx.doi.org/10.1090/surv/123

GIT94 D. Mumford, J. Fogarty, and F. Kirwan, Geometric invariant theory, third edition, Ergeb. Math. Grenzgeb. (2), vol. 34, Springer-Verlag, Berlin, 1994. http://dx.doi.org/10.1007/ 978-3-642-57916-5

Gro13 P. Gross, Tensor generators on schemes and stacks, arXiv:1306.5418.

GS15 A. Geraschenko and M. Satriano, Toric stacks II: Intrinsic characterization of toric stacks, Trans. Amer. Math. Soc. 367 (2015), no. 2, 1073-1094. http://dx.doi.org/10.1090/ S0002-9947-2014-06064-9 


\section{Anton Geraschenko and David Zureick-Brown}

Hal14 J. Hall, A relative GAGA principle for families of curves, J. Lond. Math. Soc. (2) 90 (2014), no 1, 29-48. http://dx.doi.org/10.1112/jlms/jdu012

HR14 J. Hall and D. Rydh, The Hilbert stack, Adv. Math. 253 (2014), 194-233. http://dx.doi. org/10.1016/j.aim.2013.12.002

HS04 M. Haiman and B. Sturmfels, Multigraded Hilbert schemes, J. Algebraic Geom. 13 (2004), no. 4, 725-769. http://dx.doi.org/10.1090/S1056-3911-04-00373-X

Knu71 D. Knutson, Algebraic spaces. Lecture Notes in Math., vol. 203. Springer-Verlag, Berlin, 1971.

Kre13 A. Kresch, Flattening stratification and the stack of partial stabilizations of prestable curves, Bull. Lond. Math. Soc. 45 (2013), no 1, 93-102. http://dx.doi.org/10.1112/blms/bds066

Lie06 M. Lieblich, Remarks on the stack of coherent algebras, Int. Math. Res. Not. Art. ID 75273, 2006. http://dx.doi.org/10.1155/IMRN/2006/75273

LMB00 G. Laumon and L. Moret-Bailly, Champs algébriques, Ergeb. Math. Grenzgeb. (3), Folge A Series of Modern Surveys in Mathematics, vol. 39 , Springer-Verlag, Berlin, 2000.

LO09 M. Lieblich and B. Osserman, Functorial reconstruction theorems for stacks, J. Algebra 322 (2009), no. 10, 3499-3541. http://dx.doi.org/10.1016/j.jalgebra.2009.08.009

Lur04 J. Lurie, Tannaka duality for geometric stacks, arXiv:math/0412266.

Mil80 J. S. Milne, Étale cohomology, Princeton Math. Ser., vol. 33, Princeton Univ. Press, Princeton, N.J., 1980.

Ols05 M. C. Olsson, On proper coverings of Artin stacks, Adv. Math. 198 (2005), no. 1, 93-106. http://dx.doi.org/10.1016/j.aim.2004.08.017

SGA3 Séminaire de Géométrie algébrique du Bois-Marie, 1962-1964 (SGA3), Schémas en groupes, Tome 1: Propriétés générales des schémas en groupes, dirigé par M. Demazure et A. Grothendieck, Lecture Notes in Math., vol. 151, Springer-Verlag, Berlin-New York, 1970.

SGA4 Séminaire de Géométrie algébrique du Bois-Marie, 1963-1964 (SGA4), Théorie des topos et cohomologie étale des schémas, dirigé par M. Artin, A. Grothendieck et J. L. Verdier, avec la collaboration de P. Deligne et B. Saint-Donat, Lecture Notes in Math., vols. 269, 270, and 305, Springer-Verlag, Berlin, 1973.

Sil94 J. H. Silverman. Advanced topics in the arithmetic of elliptic curves, Grad. Texts in Math., vol. 151, Springer-Verlag, New York, 1994. http://dx.doi.org/10.1007/ 978-1-4612-0851-8

SP The Stacks Project Authors, Stacks Project, available at http://math.columbia.edu/ algebraic_geometry/stacks-git.

Tot04 B. Totaro, The resolution property for schemes and stacks, J. Reine Angew. Math. 577 (2004), 1-22. http://dx.doi.org/10.1515/crll.2004.2004.577.1

Anton Geraschenko geraschenko@gmail.com

Google, 340 Main St., Venice, CA 90291, USA

David Zureick-Brown dzb@mathcs.emory.edu

Department of Mathematics \& Computer Science, Emory University, 400 Dowman Dr., W401 Atlanta, GA 30322, USA 